\title{
Inferring the zonal distribution of measured changes in the meridional overturning circulation
}

\author{
A. M. de Boer ${ }^{1}$ and H. L. Johnson ${ }^{2}$ \\ ${ }^{1}$ University of East Anglia, Norwich, UK \\ ${ }^{2}$ University of Reading, Reading, UK
}

Received: 22 September 2006 - Published in Ocean Sci. Discuss.: 10 October 2006

Revised: 10 January 2007 - Accepted: 29 January 2007 - Published: 6 February 2007

\begin{abstract}
Recently, hydrographic measurements have been used to argue that the meridional overturning circulation at $25^{\circ} \mathrm{N}$ has decreased by $30 \%$ over the last 50 years. Here we show that the most likely interpretation consistent with this approach (i.e., with the dynamic method together with a level-of-no-motion assumption and Ekman dynamics) is that any decrease in strength of the deep western boundary current must have been compensated, not by a basin-wide increase in upper layer southward flow, but by changes in the nonlinear region immediately outside of the Florida Straits.
\end{abstract}

\section{Introduction}

Coupled climate model experiments suggest that the Atlantic meridional overturning circulation may weaken in response to global warming, with significant implication for European climate. As a result, the recent claim of Bryden et al. (2005, hereafter BLC05) that the meridional overturning circulation at $25^{\circ} \mathrm{N}$ has decreased over the last 50 years, has received widespread attention. They find that a weakened deep western boundary current has been compensated by an increase in the southward flow in the upper layer of the ocean interior. We demonstrate here that, if we take the BLC05 results at face value (i.e., if we believe that they resolve the necessary temporal and spatial scales), additional information about the zonal distribution of the upper layer changes can be obtained from the theory which results from their assumptions. Note that, here and throughout our manuscript, we use the term upper layer flow to describe all the meridional transport which occurs above the reference level (defined by BLC05 at $3200 \mathrm{~m}$ in the ocean interior).

In regions devoid of mesoscale eddy activity, the assumptions made by BLC05 imply Sverdrup balance. Be-

Correspondence to: A. M. de Boer

(a.deboer@uea.ac.uk) cause the curl of the windstress has not changed significantly (H. L. Bryden, personal communication), Sverdrup balance predicts a constant upper layer meridional transport. Therefore, any changes observed by BLC05 must have occurred in regions where eddies are prevalent so that Sverdrup theory breaks down.

Sverdrup theory (Sverdrup, 1947) is by no means proven to hold even in non-eddying regions of the North Atlantic (Wunsch, 1996) and we do not assume at any stage that it does. Yet this is of no consequence to our argument. To the extent that the method employed by BLC05 and similar studies relies on the assumptions that go into Sverdrup theory, the interpretation of their results should be consistent with its predictions, whether or not the theory is valid in the real ocean.

\section{The link to Sverdrup balance}

Before we illustrate the connection between the BLC05 assumptions and Sverdrup balance, it is useful to define some key regions. The first is the northward flowing Gulf Stream (GS), confined within the Florida Straits. Following BLC05, we refer to the rest of the basin as the mid-ocean geostrophic region (MOG). Within the MOG we further define two physical regimes: a nonlinear region (NLR) where mesoscale eddies are of first order importance (which we shall argue lies on the western side of the basin, just east of the Bahamas), and a more linear region (LR) which is largely eddy-free (and spans the rest of the ocean interior).

In their analysis BLC05 make the following assumptions:

a) The mid-ocean (MOG) is in geostrophic balance to first order and, therefore, the meridional velocity is given by

$v_{g}=\frac{p_{x}}{\rho_{o} f}+\delta$,

where $p_{x}$ is the pressure gradient in the zonal direction, $\rho_{o}$ is a reference density, $f$ is the Coriolis parameter and $\delta$

Published by Copernicus GmbH on behalf of the European Geosciences Union. 
represents other terms in the momentum equation which are negligibly small. Combined with hydrostatic balance, this gives the well-known thermal wind equation on which the hydrographic method is based.

b) There exists a fixed deep reference level at which the velocity is either zero or uniform and small.

c) The physics of the top frictional layer is governed by Ekman dynamics, i.e., the net transport is at 90 degrees to the direction of the wind and is given by

$$
\frac{\tau}{\rho_{o} f} \text {, }
$$

where $\tau$ is the magnitude of the surface windstress (Ekman, 1905).

d) Other assumptions that are not directly related to our argument, for example, that the temporal resolution of the measurements is adequate to resolve the decadal variability.

Assumptions (a) to (c) lead to Sverdrup balance as follows. In regions where the spatial derivatives of the $\delta$ terms in the momentum equations are negligible (i.e., in the LR), geostrophy (assumption a) together with the conservation of mass give the linear vorticity equation:

$$
v_{g}=\frac{f w_{z}}{\beta},
$$

where $\beta$ is the meridional derivative of $f$ and $w_{z}$ is the vertical derivative of the vertical velocity (Cushman-Roisin, 1994). The assumption of a reference level, $z_{r}$, whose depth is constant in time and space and at which the velocity is negligible (assumption b) implies that the vertical velocity is zero at that level (Wunsch, 1996). Integrating Eq. (3) from $z_{r}$ to the bottom of the Ekman layer, $z_{E}$, gives an expression for the depth-integrated meridional geostrophic velocity between these levels,

$$
V_{g}=\int_{z_{r}}^{z_{E}} v_{g} d z=\frac{f w_{E}}{\beta} .
$$

Here $w_{E}$ is equal to the vertical velocity at the base of the Ekman layer and is therefore given by the divergence of the sum of the Ekman and geostrophic transports in the Ekman layer (assumption c),

$$
\begin{aligned}
w_{E} & =\nabla \times\left(\frac{\tau}{\rho_{o} f}\right) \\
& =\frac{1}{\rho_{o} f} \nabla \times \tau-\frac{\beta}{f} V_{E}-\frac{\beta}{f} V_{g e},
\end{aligned}
$$

where $V_{E}$ is the meridional Ekman transport and $V_{g e}$ is the meridional geostrophic transport in the Ekman layer. (For further details see, for instance, Stewart, 2005.) Note that we have followed the standard convention of referring to the depth-integrated Ekman and Sverdrup velocities as transports. In fact, they must be horizontally integrated to represent true volume transport. Combining Eq. (4) and Eq. (5) now gives the familiar Sverdrup transport

$$
V=V_{g}+V_{g e}+V_{E}=\frac{1}{\rho_{0} \beta} \nabla \times \tau .
$$

Because assumptions (a) to (c) lead directly to Eq. (6) in the LR, their use implies that Sverdrup balance also holds there. Sverdrup balance dictates that if the windstress does not change, then the total transport above the reference level must not change either. Since BLC05 find that the Ekman transport has not changed, and neither has the windstress curl during the last 50 years, the geostrophic transport in the LR should also be constant. Any change in the MOG transport must therefore manifest itself in the NLR.

It is possible for the flow in the NLR to be geostrophic to leading order (i.e. the $\delta$ terms are negligible in Eq. 1), and yet for the spatial derivatives of the $\delta$ terms to result in a nonnegligible contribution to the vorticity balance. This occurs when the Rossby number $\mathrm{U} /(\mathrm{fL}) \ll 1$, but $\mathrm{U} /\left(\beta \mathrm{L}^{2}\right) \gtrsim 1$, where $\mathrm{U}$ and $\mathrm{L}$ are typical horizontal velocity and length scales. This parameter space describes regions of mesoscale eddy activity $\left(\mathrm{L} \sim 10 \mathrm{~km}, \mathrm{U} \sim 10 \mathrm{~cm} \mathrm{~s}^{-1}\right)$, in which relative rather than planetary vorticity dominates (see a standard oceanographic text such as Pedlosky (1987) for further details). It is in this regime that any changes in upper layer southward transport found by BLC05 must have occurred. At $25^{\circ} \mathrm{N}$ the only region dominated by small scale features is on the western side of the basin, just east of the Bahamas. This region is part of the western boundary current system and mesoscale eddies are common here (Lee et al., 1996). In contrast, away from the western boundary eddy kinetic energy is low (Ducet et al., 2000) so that linear vorticity balance is a good approximation and the southward upper layer flow cannot have changed. From here on we refer to the LR, not only generally as a region devoid of eddies, but explicitly as the ocean interior away from the nonlinear western boundary region.

For simplicity, we have presented our argument so far as concisely as possible. A few additional comments about the finer details are appropriate. First, because the windstress fields over the last 50 years have a large uncertainty associated with them, it is possible that the wind stress curl has in fact increased, leading to a larger southward $V$ in the LR. If the increased Sverdrup transport is not negated by compensating changes in the NLR or GS region, the net result would indeed indicate a weakened meridional overturning circulation.

Second, in the LR, the application of a reference level uniform in time and space is based on the implicit assumption that all velocity components are vanishingly small there. BLC05 adds a constant meridional reference level velocity across the basin to conserve mass. However, because the vertical shear and hence the isopycnal slope are assumed small at the reference level, this adjustment does not affect the assumption that $w=0$ there, required for the above derivation of the Sverdrup transport (Wunsch, 1996). (This is not true at the shallower reference level within the NLR, where there is considerable vertical shear due to the presence of the deep western boundary current.) Physically, Sverdrup balance expresses the fact that the meridional velocity of a fluid column stretching from $z_{r}$ to the bottom of the Ekman layer is determined by the extent to which it is stretched or compressed in the vertical (in order to conserve total vorticity). For zero 
vertical velocity at the base of the column and a fixed vertical velocity at the top, the meridional flow should be unchanged. Note that any non-zero vertical velocity at the ocean floor is irrelevant to our argument.

Finally, we consider the effect of transient features such as Rossby waves. Because BLC05 claim to resolve the relevant time scales (assumption d), we too can neglect variablility on time scales shorter than annual in our analysis. However, long and short baroclinic Rossby waves have time scales longer than this. The energy in short Rossby waves (with wavelengths shorter than the Rossby deformation radius, $\mathrm{L}_{d}$ ) travels eastward from the western boundary and is dissipated within our nonlinear western boundary region. In contrast, long Rossby waves (with wavelengths longer than $\mathrm{L}_{d}$ ) are seen throughout the basin, but are not important in the vorticity budget. Therefore, as with the mesoscale variability, Rossby waves can only significantly affect the vorticity budget in the NLR.

\section{Conclusions}

We have demonstrated here that it is impossible to arrive at a variable upper layer transport in the ocean interior using the assumptions employed by BLC05. We have not assumed at any stage that Sverdrup balance holds in the North Atlantic, and our intention was certainly not to refute or support it. Rather, we have shown that the assumptions implicit in the BLC05 method lead to Sverdrup balance in linear regions. The only interpretation of their results is, therefore, that any compensation for a weakening of the deep western boundary current (if it has indeed weakened) must have occurred in the NLR where relative vorticity becomes important and Sverdrup theory breaks down. At $25^{\circ} \mathrm{N}$ only the western boundary region exhibits enough small scale eddies to satisfy this criterion. BLC05 are somewhat ambiguous about where the upper ocean changes occur. On the one hand, they point out that southward flow is certainly stronger in the western boundary region in 1998 and 2004 (see their supplementary Fig. 1). On the other hand, they claim that the increased southward flow in the MOG is not due to variability in the western boundary region alone, because it is accompanied by a general warming on the western side of the basin that leads to a steeper slope of the thermocline right across the Atlantic. This, together with the assumption of a fixed level-of-nomotion, is taken to imply that the overall southward transport in the interior has increased. We have shown that this second interpretation can be rejected. The emerging picture is therefore one in which upper layer changes of the meridional overturning circulation are manifested in a weakened northward western boundary current system (GS + NLR) and constant southward interior transport. This could have significant implications for the heat transport across $25^{\circ} \mathrm{N}$, as well as for monitoring strategies, since it suggests that resources should perhaps be concentrated on the western side of the basin.
Hydrographic data, such as that used by BLC05, provide more useful information about the time-dependent overturning circulation when combined with additional constraints. These may come from current meter measurements of the reference velocity, or from satellite altimetry measurement of sea surface slope. In practice, changes in the Atlantic meridional overturning circulation will be determined using a data assimilation approach that combines all available data, including the RAPID monitoring array deployed at $26.5^{\circ} \mathrm{N}$.

Acknowledgements. We would like to thank R. Tailleux, D. Marshall and D. Nof for insightful comments on an earlier draft of the manuscript, and C. Wunsch for a useful discussion. H. L. Johnson is funded under a Royal Society University Research Fellowship and A. M. de Boer gratefully acknowledges support from NERC Quatenary Quest fellowship NE/D001803/1.

Edited by: S. M. Griffies

\section{References}

Bryden, H. L., Longworth, H. R., and Cunningham, S. A.: Slowing of the Atlantic meridional overturning circulation at 25 degrees N, Nature, 438, 655-657, 2005.

Cushman-Roisin, B.: Introduction to Geophysical Fluid Dynamics, Prentice Hall, 1994.

Ducet, N., Le Traon, P. Y., and Reverdin, G.: Global high-resolution mapping of ocean circulation from the combination of T/P and ERS-1/2, J. Geophys. Res., 105, 19477-19498, 2000.

Ekman, V. W.: On the influence of the earth's rotation on ocean currents, Ark. Mat. Astron. Fys., 2, 1-53, 1905.

Lee, T. L., Johns, W. E., Zantopp, R. J., and Fillenbaum, E. R.: Moored Observations of Western Boundary Current Variability and Thermohaline Circulation at $26.5^{\circ}$ in the Subtropical North Atlantic, J. Phys. Oceanogr., 26, 962-983, 1996.

Pedlosky, J.: Geophysical Fluid Dynamics, Springer, 710 pp, 1987.

Stewart, R. H.: Introduction to Physical Oceanography, http: //oceanworld.tamu.edu/resources/ocng_textbook/contents.html, 2005.

Sverdrup, H. U.: Wind-Driven Currents in a Baroclinic Ocean with Application to the Equatorial Currents of the Eastern Pacific, Proceedings of the National Academy of Sciences of the United States of America, 33, 318-326, 1947.

Wunsch, C.: The Ocean Circulation Inverse Problem, Cambridge University Press, 443 pp, 1996. 